\title{
Botulinum Toxin Treatment on Upper Limb Function in School Age Children With Bilateral Spastic Cerebral Palsy: One Year Follow-up
}

\author{
Jee Sun Lee, MD, Kyu Bum Lee, MD, Yu Ryun Lee, MD, You Nam Choi, MD, \\ Chul Woo Park, MD, Sang Duck Park, Dong Hwa Jung, PhD, Chul Sang Lee \\ Department of Rehabilitation Medicine, Seoul Rehabilitation Hospital, Seoul, Korea
}

Objective To prospectively investigate the long-term effects of botulinum toxin treatment on the upper limb function and performance of school age children with spastic bilateral cerebral palsy, who have limitations in performing activities of daily living and school activities, due to spasticity of the upper extremities.

Methods Botulinum type A toxin (BoNT-A) was injected into 24 spastic upper limbs of 15 children. We used a Modified Ashworth Scale and a Modified Tardieu Scale for the evaluation of upper limb spasticity, and Quality of Upper Extremity Skills Test (QUEST), Canadian Occupational Performance Measure (COPM), and Test of VisualMotor Skills-Revised (TVMS-R) for the evaluation of upper limb function and performance.

Results Upper limb spasticity continuously decreased until the end of the one-year follow-up. Upper limb function on QUEST and COPM showed the best performance at 3 months and deteriorated slightly, but still showed a significantly better performance at 9 and 12 months than at pre-injection. In more functional nine subjects who could perform TVMS-R, the performance enhancement effects remained constant after 12 months, suggesting that the reduced spasticity led to the learning effect acquired by the repeated use of the affected upper limb.

Conclusion For school age children with bilateral spastic cerebral palsy whose upper limb functions are important, BoNT-A injections seem to be of help in the performance of school activities and activities of daily living.

Keywords Cerebral palsy, Botulinum A toxin, Upper limb, School

Received December 15, 2011; Accepted October 11, 2012

Corresponding author: Jee Sun Lee

Department of Rehabilitation Medicine, Seoul Rehabilitation Hospital, 30 Galhyeon-ro 11-gil, Gusan-dong, Eunpyeong-gu, Seoul 122-822, Korea Tel: +82-2-6020-3001, Fax: +82-2-6020-3009, E-mail: jslee704@hanmail. net

(c) This is an open-access article distributed under the terms of the Creative Commons Attribution Non-Commercial License (http://creativecommons. org/licenses/by-nc/3.0) which permits unrestricted noncommercial use, distribution, and reproduction in any medium, provided the original work is properly cited.

Copyright () 2013 by Korean Academy of Rehabilitation Medicine

\section{INTRODUCTION}

Cerebral palsy (CP) is one of the most common developmental disorders in childhood, affecting the entire life cycle. CP children require treatment and management plans according to the developmental tasks they face, throughout their whole life cycle. Spasticity of CP interrupts longitudinal muscle growth, decreases muscle volume, and eventually shortens spastic muscles [1]. Children with bilateral spastic CP frequently have spas- 
ticity in the upper extremities. Spasticity and increased muscle tone with associated reactions cause limitation of activity and restrict participation in various activities [2]. All these problems become prominent in school age children, who need more independence in several domains, such as in activities involved in learning and applying knowledge, general tasks, mobility, and self-care.

It was noted that treatment of spasticity is important in the management of $\mathrm{CP}$ [3]. Botulinum type A toxin (BoNT-A) injection is a major method of treatment for managing spasticity of the upper extremities. The ultimate aim of the BoNT-A injection in children with CP is to enhance motor learning and achieve a greater functional gain through the reduction of tone in the injected muscles [4]. Studies on the effects of BoNT-A injection on spastic upper limb of CP children used various evaluation tools and reported varying results accordingly [3,5-8].

The purpose of this study was to prospectively investigate the long-term effects of BoNT-A injection on the upper limb function and performance of activities in school age children with spastic bilateral $\mathrm{CP}$, who have limitations in school and home activities, due to spasticity of the upper extremities.

\section{MATERIALS AND METHODS}

\section{Subjects}

Fifteen children with bilateral spastic type $\mathrm{CP}$, who were of school age and capable of maintaining the sitting position on a chair or wheelchair, and had any form of hand grip, despite spasticity in the favored upper limb, as well as those available for the one-year follow-up were enrolled in this study. Children with a history of past botulinum toxin or phenol injection, or orthopedic surgery on the upper limb were excluded from the study. Any medication or surgery, including neurologic or ophthalmic surgery, which might influence the spasticity or study result, was not allowed. Nine subjects were injected in the targeted muscles of the bilateral upper limbs, due to the spasticity of both upper limbs in these subjects. Additionally, six other subjects were injected in the unilateral upper limb because they had spasticity only on one side, or spasticity of the other limb did not deteriorate significantly in its function despite bilateral spasticity.

The mean age of the subjects was $11.4 \pm 2.3$ years ( 8 male, 7 female). The subjects participated in this study had worse gross motor skills compared to subjects in other publications. Ten out of the 15 subjects were categorized as Gross Motor Function Classification System (GMFCS) level IV, and three out of 15 were level V. Only one subject was evaluated as gross motor function level I, which is the best level, and one as level II. For upper limb function, three subjects were classified into Manual Ability Classification System (MACS) level II, six subjects into level III, and another six into level IV. The deformity they had were as follows: limited shoulder flexion of 11 limbs, limited shoulder abduction of 3 limbs, limited shoulder external rotation of 10 limbs, limited elbow extension of 8 limbs, limited supination of 14 limbs, swan neck deformity of 3 limbs, limited finger extension of 2 limbs, and thumb in palm of 23 limbs.

\section{Methods}

\section{Intervention}

BoNT-A (Botox; Allergan Inc., Irvine, CA, USA), was injected in 24 spastic upper limbs of $15 \mathrm{CP}$ children for this one-year prospective longitudinal study. The muscles were selected complying with upper limb function goals, which are requested by the patients and guardians based on their Canadian Occupational Performance Measure (COPM) reports. Spastic muscles that inhibit normal functioning were selected, and a predefined dose of Botox was injected depending on the degree of spasticity. BoNT-A was diluted with $1 \mathrm{cc}$ of $0.9 \%$ saline. EMLA cream (AstraZeneca, Kings Langley, UK), a lidocaine-based local anesthetic, was applied on the injection site before injection.

A neuromuscular stimulator (CLAVIS; Medtronic Inc., Minneapolis, MN, USA) was used to find a motor point by applying $1-2 \mathrm{~mA}$ of stimulus for $100 \mathrm{~ms}$ before injection. Subjects received a single injection of BoNT-A during the study period. Other procedures or surgery that might affect the spasticity were not allowed during this period. Nine subjects received an injection in both upper limbs and six subjects received injections in only one side. The total dose of BoNT-A was 134.2 \pm 51.1 units on average, and the mean dose per weight was $5.4 \pm 2.1$ units $/ \mathrm{kg}$. An average of nine (5-13) muscles were injected per subject, including $5.4(2-8)$ muscles per limb. Subjects received injections most often in the adductor pollicis, followed by the pronator teres, brachialis, flexor carpi radialis and ulnaris, and teres major muscle (Table 1). 
Table 1. Muscles injected for management of spasticity

\begin{tabular}{lc}
\hline \multicolumn{1}{c}{ Muscle } & No. of limbs $(\mathbf{n = 2 4 )}$ \\
\hline Adductor pollicis & 23 \\
Pronator teres & 21 \\
\hline Brachialis & 18 \\
\hline Flexor carpi radialis & 18 \\
Flexor carpi ulnaris & 18 \\
Teres major & 17 \\
\hline Pectoralis major & 11 \\
\hline Pronator quadratus & 2 \\
\hline Flexor digitorum profundus & 2 \\
\hline Infraspinatus & 1 \\
\hline Flexor pollicis longus & 1 \\
\hline
\end{tabular}

\section{Outcome measurements}

Modified Ashworth Scale and Modified Tardieu Scale were measured to confirm the spasticity in large muscles, such as the shoulder flexor, internal rotator, elbow flexor, forearm pronator, and wrist flexor of each upper limb. The hand intrinsic muscles were excluded. The Modified Ashworth Scale measures from 0 (normal with no resistance to movement) to grade 1 , grade 1.5 , grade 2 , grade 3 , and grade 4 (maximum spasticity making passive movements extremely difficult), and each grade was recorded by scores for the purpose of analysis, with grade 1.5 equaling a score of 1.5. R1 (angle of catch) and R2 (passive end range) were measured by the Modified Tardieu Scale to calculate the effect of spasticity (R2-R1, dynamic component). The degree of spasticity was checked before the injection, and at 2 weeks, 3, 6, 9, and 12 months after the injection.

Outcome measures included upper limb function, activity performance and satisfaction. Upper limb function was evaluated using the Quality of Upper Extremity Skills Test (QUEST), developed for the qualitative evaluation of upper limb movements in CP children, and it is a reliable measurement tool for estimating the treatment effects on motor ability and function in CP children [9]. Improvement of performance and functional goal attainment were evaluated using a COPM. COPM was performed by interviewing patients and their guardians to identify the major disabilities and needs in daily life, school life, and leisure activities of the subjects. Changes in the performance of the revised Test of Visual-Motor Skills (TVMS-R) in 9 patients, who had more functional upper limbs and could handle a pencil, were recorded. It calculates a converted motor age based on the TVMS-R scale, reflecting upper limb functioning in school activities. The TVMS-R [10] evaluates eight performance classifications, thereby enabling direct evaluation of learning skills and performance areas of school age children. These outcome measures were evaluated before the injection, two weeks after the injection when BoNT-A reaches its maximum effect, 3 months after the injection as an average duration of the effect, and at 9 and 12 months to determine the long-term effects of botulinum toxin treatment. An occupational therapist, in charge of school age children, performed these evaluations.

\section{Data analysis}

SPSS ver. 15.0 (SPSS Inc., Chicago, IL, USA) was used for statistical analysis. Demographic and clinical data of the study group was used for descriptive statistics. The changes of spasticity in the joint, where the injected muscles act, were analyzed.

A paired t-test was performed for the Modified Ashworth Scale and Modified Tardieu Scale, respectively, to compare the changes of pre- and post-treatment muscle tones. Immediate and long-term effects on function and performance were compared with the baseline values, using a paired t-test $(\mathrm{p}<0.05)$.

\section{RESULTS}

\section{Changes in impairment}

Scores of the Modified Ashworth Scale, which is an assessment tool for spasticity, decreased with statistical significance at 2 weeks, 3, 6, 9, and 12 months after the injection, compared to the baseline, for shoulder flexion, internal rotation, elbow flexion, forearm pronation and wrist flexion; however, the decrease was not statistically significant for shoulder flexion at 12 months, shoulder internal rotation at 9 and 12 months, and wrist flexion at 12 months. Generally, spasticity was reduced significantly at two weeks and increased consistently thereafter, but the reduction of spasticity from the base line was maintained until 12 months (Table 2).

Based on the Modified Tardieu Scale result, R1 was increased. There was significant decrease of the R2-R1 value of elbow flexion, forearm pronation, and wrist flexion at 2 weeks, 3, 6, 9, and 12 months after the injection, 
Table 2. Changes of Modified Ashworth Scale $(n=24)$

\begin{tabular}{lcccccc}
\hline & Pre-injection & After 2 wk & After 3 mo & After 6 mo & After 9 mo & After 12 mo \\
\hline Shoulder flexion & $1.18 \pm 0.34$ & $0.82 \pm 0.40^{*}$ & $0.75 \pm 0.46^{*}$ & $0.86 \pm 0.38^{*}$ & $0.571 \pm 0.535^{* *}$ & $0.80 \pm 0.45$ \\
$\begin{array}{l}\text { Shoulder internal } \\
\text { rotation }\end{array}$ & $1.43 \pm 0.37$ & $1.067 \pm 0.372^{* *}$ & $1.14 \pm 0.23^{*}$ & $1.056 \pm 0.167^{* *}$ & $1.23 \pm 0.56$ & $1.25 \pm 0.71$ \\
Elbow flexion & $1.42 \pm 0.43$ & $0.556 \pm 0.511^{* * *}$ & $0.893 \pm 0.525^{* *}$ & $0.850 \pm 0.474^{* *}$ & $0.900 \pm 0.387^{* * *}$ & $1.25 \pm 0.35^{*}$ \\
Forearm pronation & $1.57 \pm 0.50$ & $0.705 \pm 0.504^{* * *}$ & $0.844 \pm 0.569^{* * *}$ & $0.833 \pm 0.577^{* *}$ & $0.972 \pm 0.629^{* *}$ & $1.23 \pm 0.53^{*}$ \\
Wrist flexion & $1.20 \pm 0.57$ & $0.400 \pm 0.503^{* * *}$ & $0.333 \pm 0.488^{* * *}$ & $0.63 \pm 0.50^{*}$ & $0.82 \pm 0.53^{*}$ & $1.08 \pm 0.47$ \\
\hline
\end{tabular}

Values are presented as mean \pm standard deviation.

${ }^{*} \mathrm{p}<0.05,{ }^{* *} \mathrm{p}<0.01,{ }^{* * *} \mathrm{p}<0.001$.

Table 3. Changes of Modified Tardieu Scale (R2-R1; n=24)

\begin{tabular}{|c|c|c|c|c|c|c|}
\hline & Pre & Aft & Aft & Aft & $9 \mathrm{mo}$ & $12 \mathrm{mo}$ \\
\hline Shoulder flexion & $51.00 \pm 50.87$ & $10.00 \pm 9.43^{*}$ & $15.00 \pm 9.26$ & $24.29 \pm 19.02$ & $20.00 \pm 23.09 *$ & 23.75 \\
\hline $\begin{array}{l}\text { ler internal } \\
\text { on }\end{array}$ & .86 & $24.000 \pm$ & 39.0 & 27.7 & 39.0 & 45. \\
\hline Elbow flexion & $59.69 \pm 19.10$ & $7.500 \pm 10.646^{* * *}$ & $35.00 \pm 25.05^{*}$ & $25.625 \pm 22.903^{* *}$ & $36.92 \pm 23.85^{*}$ & $37.50 \pm 12.82^{*}$ \\
\hline $\begin{array}{l}\text { Forearm } \\
\text { pronation }\end{array}$ & $74.05 \pm 31.61$ & $14.762 \pm 11.233^{* * *}$ & $23.333 \pm 16.330^{* *}$ & *34.091 $\pm 29.901^{* *}$ & $38.824 \pm 29.130^{* * *}$ &  \\
\hline Wrist flexion & $52.00 \pm 35.81$ & $4.000 \pm 7.539 * * *$ & $8.667 \pm 15.055^{* *}$ & ${ }^{*} 15.000 \pm 15.076^{* *}$ & $22.941 \pm 21.144^{* *}$ & $34.583 \pm 20.500^{* *}$ \\
\hline
\end{tabular}

Values are presented as mean \pm standard deviation.

R1, angle of catch on Modified Tardieu Scale; R2, passive end range on Modified Tardieu Scale.

${ }^{*} \mathrm{p}<0.05,{ }^{* *} \mathrm{p}<0.01,{ }^{* * *} \mathrm{p}<0.001$.

Table 4. Changes of upper extremity function and performance

\begin{tabular}{lrccccc}
\hline & No. & Pre-injection & After 2 wk & After 3 mo & After 9 mo & After 12 mo \\
\hline QUEST total score & 15 & $36.12 \pm 34.07$ & $43.057 \pm 33.446^{* * *}$ & $51.472 \pm 25.645^{* * *} 48.250 \pm 27.905^{* *}$ & $37.615 \pm 37.663^{* *}$ \\
COPM performance score & 15 & $3.84 \pm 1.21$ & $4.633 \pm 1.522^{* *}$ & $5.223 \pm 1.473^{* * *}$ & $5.025 \pm 1.617^{* *}$ & $4.87 \pm 1.60^{*}$ \\
COPM satisfaction score & 15 & $3.28 \pm 1.34$ & $4.740 \pm 1.498^{* * *}$ & $5.346 \pm 1.641^{* * *}$ & $4.892 \pm 1.498^{* *}$ & $4.25 \pm 1.74^{*}$ \\
TVMS-R motor age (mo) & 9 & $53.33 \pm 7.84$ & $57.444 \pm 7.699^{* *}$ & $61.222 \pm 8.800^{* *}$ & $61.250 \pm 9.867^{* *}$ & $64.67 \pm 14.43^{*}$ \\
\hline
\end{tabular}

Values are presented as mean \pm standard deviation.

QUEST, Quality of Upper Extremity Skills Test; COPM, Canadian Occupational Performance Measure; TVMS-R, Test of Visual Motor Skills-revised.

${ }^{*} \mathrm{p}<0.05,{ }^{* *} \mathrm{p}<0.01,{ }^{* * *} \mathrm{p}<0.001$.

indicating that the BoNT-A injection was significantly effective in reducing spasticity in these areas. A decrease in the R2-R1 value, compared with before the injection remained at every follow-up examination after the injection; however, the decrease was not statistically significant at several evaluation times for shoulder flexion and shoulder internal rotation (Table 3).

Changes in the upper limb function and performance

Based on the results of upper limb function and performance which were compared before and after the injection, both QUEST and COPM showed the highest scores at 3 months after the injection. The total score of QUEST, which reflects upper limb functioning, and both the performance and satisfaction scores of COPM, which reflect activity performance, peaked with statistical significance at 3 months after the injection $(p<0.001)$. The scores declined at 9 months and 12 months; however, the improvement of QUEST $(\mathrm{p}<0.01)$ and COPM $(\mathrm{p}<0.05)$ was statistically significant, compared with the scores before injection. The satisfaction score of COPM increased more than the performance score at 2 weeks and 3 months af- 
ter the injection compared to pre-injection. In addition, the satisfaction score of COPM decreased more than the performance score between 9 and 12 months. TVMS$\mathrm{R}$ was performed in nine patients with relatively more functional upper limbs to evaluate the visual-motor skills required for school activities. The mean motor age improved reasonably from 53.33 months before injection to 57.44 months ( $\mathrm{p}<0.01), 61.22$ months $(\mathrm{p}<0.01), 61.25$ months $(\mathrm{p}<0.01)$, and 64.67 months $(\mathrm{p}<0.05)$ at 2 weeks, 3,9 , and 12 months of the study, respectively; and the pattern showed a gradual rise until 12 months, which was a different result from that obtained with the other function scales (Table 4 ).

\section{DISCUSSION}

Since botulinum toxin was firstly introduced as a treatment for cerebral palsy in 1993, it has become popular throughout the world as a method of therapy for this disorder [11]. A number of studies reported the effect of BoNT-A injections in cerebral palsy children [3]. Many previous articles reporting the effects of BoNT-A on upper limb function focused on subjects with better gross motor functional level than the subjects in this study, and with a follow-up period of less than 6 months $[3,4,12,13]$. Only a few studies performed long-term follow-up of more than one year [14]. The literature review for BoNT-A injection on the upper limbs of cerebral palsy patients could not find consistent results about the effect of BoNT-A injections, and found confounding factors [3]. In addition, an optimal age for dynamic motor development when most potential motor skills can be obtained was suggested by some researchers. In this review, preschool children, the age from four to six, was reported to be most suitable for BoNT-A injections if the child had the motivation to use the affected parts in performing activities of daily living (ADL). Another systemic review reported that a combination of BoNT-A and occupational therapy is more effective than occupational therapy alone in reducing impairment, improving the activity level outcomes and attaining goal achievement [15]. The reviewers recommended that BoNT-A should not be used in isolation, but should be combined with a well-organized program of occupational therapy. Recently, a published systemic review and meta-analysis of the therapeutic management of upper limb dysfunction in children with congenital hemiplegia investigated the therapeutic effects of nonsurgical intervention in a survey of twelve studies and seven systemic reviews [16]. No one treatment approach seems to be superior over the other; however, injections of BoNT-A provide a supplementary benefit in a variety of upper-limb training approaches, such as constraint-induced movement therapy, hand-arm bimanual intensive training, and neurodevelopment therapy. The analyzed studies in this article showed a wide age range of subjects, from 0 to 19 years old. Those previously published articles regarding the effects of BoNT-A on upper limbs dealt with mostly younger children. No study that investigated solely school aged children has been published and no study focusing on subjects with bilateral spastic cerebral palsy has been reported on.

This study on the effect and duration of BoNT-A for treating the spasticity of the upper limbs restricted subjects to only those with bilateral spastic cerebral palsy and older school age children because the hemiplegic group and preschool age children have been previously investigated in many articles $[3,17]$. The mean age of our subjects in this study was 11.4 years, and ages ranged from 8.7 to 15.6 years old. This study indicates that school age children and teenagers could also benefit from BoNT-A injections, with improvement in the domains of upper limb functioning and performance of tasks as well as actions. In addition, it was encouraging that the effects of injection lasted throughout the one-year follow-up.

Many current articles about school age children and teenagers with $\mathrm{CP}$ reported that gross motor development regressed in these people. The study in 2009 about the natural history of gross motor development in CP noted that children with $\mathrm{CP}$, who were categorized into GMFCS III, IV, and V, have a peak of function around 6-8 years of age and prominent regression of motor function follows after that time [18]. Also gross motor skills can get worse due to muscle contracture when children with bilateral spastic CP enter school, because they usually experience rapid growth during this time, and besides, they usually sit on a chair for long periods of time once they enter school [19]. In this study, thirteen out of fifteen subjects belonged to GMFCS IV and V, which they attained at the age of gross motor regression.

Students with limited movement skills might experience serious deterioration in their quality of life, and as a result, the performance capability of their upper limbs in 
doing ADLs is very important in determining their quality of life. Therefore, helping these children to develop skills in ADLs (getting dressed, self-toileting, and feeding), and in recreational activities and educational activities is extremely important in school age children and adolescents [20].

It was pointed out that fine motor functions, including upper-limb speed, dexterity and visual-motor control, were the most important factors associated with healthrelated quality-of-life (QOL) in ambulatory subjects with cerebral palsy [21]. Considering all these aspects, when school age children with bilateral spastic CP experience deterioration of ambulation and function, due to progressed deformity of the lower limbs, their upper limb function for ADLs performance is significant for QOLs assessment. Thus, the effectiveness of BoNT-A treatment that was seen, including advancement of upper limb function in the school age children, is presumed to be meaningful. However, BoNT-A injections for spasticity have not generally been used as a therapy for CP in school age children. Since school age children and teenagers have a strong desire and requirement for adequate upper limb functioning, as this can influence their QOL, it is reasonable to attempt to improve upper limb functioning and prevent fixed contractures in school age children with more aggressive management, such as the use of BoNT-A injections.

In this study, the subjects actively participated in the rehabilitation treatment. First of all, during an interview for COPM, the subjects selected the activities they wanted to perform as part of the treatment evaluation, and they were expected to perform the task, to set performance goals and find their limitations while pursuing the ADL. The subjects chose the items of dressing, eating using tools without spilling, using a computer mouse, fast writing in tiny letters, bimanual activities in the restroom and pushing wheelchairs, measuring the importance score of these items, with a mean score of 8.3 out of 10 . Along these lines, the school age children showed they had a strong desire for executing ADLs; therefore, improvement in upper limb functioning and in the performance of skills involved in the ADLs is imperative.

The change of spasticity and improvement in performance in this study showed different patterns during the one-year follow-up after the BoNT-A injection. The degree of spasticity, which was evaluated by a Modified Ash- worth Scale and Modified Tardieu Scale, was the lowest at two weeks after the injection, and spasticity gradually increased from 3 months after the injection. Meanwhile, performance of activities, which was evaluated by QUEST and COPM, improved the most at 3 months, and not two weeks after the injection. The explanation for this could be that the reduced spasticity enabled patients to experience and practice good mobility, inducing a learning effect in these subjects, which was manifested as enhanced performance at the 3 months follow-up, although the spasticity had already started to return. The statistically significant changes of QUEST and COPM compared to the baseline were maintained until 12 months, although the improvement diminished at 9 months after the injection. The motor age of TVMS- $R$, however, showed a gradual improvement at nine and 12 months. This tendency reflects the characteristics of this test tool. QUEST evaluates both quantitative and qualitative aspects of the upper limbs, but is more focused on the performance components of tasks, such as dissociated movement, excepting grasp, rather than the task performance itself. TVMS-R, however, directly evaluates the motor skills that could be considered as a performance area of school age children. When the spasticity was significantly reduced after BoNTA injection, the performance components of QUEST, such as dissociated movement or weight bearing, might have improved with the ability of the children to produce more smooth movements, but these improvements were evaluated as reduced from 9 months when the spasticity returned. COPM evaluates the performance area, which is scored by the subjects (or guardians) themselves. The performance score and satisfaction score in COPM were reduced again after 9 months, and the reduction of the performance score from the peak point was less than the reduction in the satisfaction score. It is possible that patients, who had already experienced reduced spasticity after the injection, might have felt more dissatisfied by the time the spasticity returned, even though their actual performance had not decreased much. Although muscle spasticity began to return after 3 months, children who had experienced a better performance pattern when the spasticity declined, gained gradual performance enhancement by repeatedly practicing their writing, which is a repetitive task in school, leading to a better outcome on TVMS-R at a later time. The results suggest that the reduced spasticity attained with BoNT treatment combined 
with continuous upper limb use in performing ADLs and school activity, might lead to continuously improved actual performance. A raw score of performance skills was converted into a motor age of TVMS-R. The motor age of TVMS-R was not adjusted for chronological age, and the learning effects of repetitive tests were not clearly manifested. The mean chronological age was 11.4 years old and the motor age was 53.33 months on average before injection. At 12 months after the injection, motor age progressed to 64.67 months. The progression was expected from the effects of the treatment because the motor age was considerably delayed before treatment, and the advancement was remarkable during the one year of the study; thus, the advancement cannot be due to chronological aging.

The results showed that despite the different patterns of improvement as shown by the assessment tools, there was decreased spasticity below the elbow joint, and the functional progression showed statistical significance compared to the baseline until 12 months later, in spite of the functional progression dwindling after the return of spasticity.

Previously, the optimal age expected for maximal effectiveness of BoNT-A treatment was younger than the age of the present subjects or was preschool age. Moreover, this study concludes that school age children and teenagers will benefit from the injections and show satisfaction with the treatment because they have a strong desire and requirement for improved upper limb functioning. Furthermore, the frequent use of their upper limbs in performing ADLs and school activities brings about repetitious training of their muscles and enhanced learning. Therefore, more active treatment with BoNT-A is considered to contribute to the improvement of QOLs in ADLs, school and social activities by advancing upper limb function in school age children.

This study has several limitations: small sample size, lack of controls, and exclusion of the measurement of spasticity in the adductor pollicis. Another limitation is that the effect of occupational therapy was not eliminated by a control study. More randomized control studies on the effects of repeated BoNT injections on the degree of functional improvement and various combined intensive rehabilitation therapies are necessary.

In conclusion, botulinum toxin treatment in school age children remained effective after 12 months, causing per- sistent upper limb performance enhancement, despite a slight return of spasticity. For older school age subjects with bilateral spastic $\mathrm{CP}$, with poor gross motor function, whose upper limb function is crucial for functional requirement and satisfaction, more active treatment of upper limb spasticity is recommended.

\section{CONFLICT OF INTEREST}

No potential conflict of interest relevant to this article was reported.

\section{REFERENCES}

1. Miller F. Cerebral palsy. New York: Springer; 2005.

2. Barnes MP, Johnson GR. Upper motor neurone syndrome and spasticity: clinical management and neurophysiology. 1st ed. Cambridge: Cambridge University Press; 2001.

3. Park ES, Rha DW. Botulinum toxin type A injection for management of upper limb spasticity in children with cerebral palsy: a literature review. Yonsei Med J 2006;47:589-603.

4. Park ES, Rha DW, Lee JD, Yoo JK, Chang WH. The short-term effects of combined modified constraintinduced movement therapy and botulinum toxin injection for children with spastic hemiplegic cerebral palsy. Neuropediatrics 2009;40:269-74.

5. Fehlings D, Novak I, Berweck S, Hoare B, Stott NS, Russo RN; Cerebral Palsy Institute. Botulinum toxin assessment, intervention and follow-up for paediatric upper limb hypertonicity: international consensus statement. Eur J Neurol 2010;17 Suppl 2:38-56.

6. Wall SA, Chait LA, Temlett JA, Perkins B, Hillen G, Becker P. Botulinum A chemodenervation: a new modality in cerebral palsied hands. Br J Plast Surg 1993;46:703-6.

7. Corry IS, Cosgrove AP, Walsh EG, McClean D, Graham HK. Botulinum toxin A in the hemiplegic upper limb: a double-blind trial. Dev Med Child Neurol 1997;39:185-93.

8. Fehlings D, Rang M, Glazier J, Steele C. An evaluation of botulinum-A toxin injections to improve upper extremity function in children with hemiplegic cerebral palsy. J Pediatr 2000;137:331-7.

9. Haga N, van der Heijden-Maessen HC, van Hoorn JF, 
Boonstra AM, Hadders-Algra M. Test-retest and interand intrareliability of the quality of the upper-extremity skills test in preschool-age children with cerebral palsy. Arch Phys Med Rehabil 2007;88:1686-9.

10. Gardner MF. Test of visual-motor skills-revised (TVMS-R). Hydesville: Psychological and Educational Publications Inc.; 1995.

11. Koman LA, Mooney JF 3rd, Smith BP, Goodman A, Mulvaney T. Management of spasticity in cerebral palsy with botulinum-A toxin: report of a preliminary, randomized, double-blind trial. J Pediatr Orthop 1994;14:299-303.

12. Rameckers EA, Duysens J, Speth LA, Vles HJ, SmitsEngelsman BC. Effect of addition of botulinum toxinA to standardized therapy for dynamic manual skills measured with kinematic aiming tasks in children with spastic hemiplegia. J Rehabil Med 2010;42:332-8.

13. Lowe K, Novak I, Cusick A. Low-dose/high-concentration localized botulinum toxin A improves upper limb movement and function in children with hemiplegic cerebral palsy. Dev Med Child Neurol 2006;48:170-5.

14. Satila H, Kotamaki A, Koivikko M, Autti-Ramo I. Upper limb function after botulinum toxin A treatment in cerebral palsy: two years follow-up of six cases. Pediatr Rehabil 2006;9:247-58.

15. Hoare BJ, Wallen MA, Imms C, Villanueva E, Rawicki HB, Carey L. Botulinum toxin A as an adjunct to treat- ment in the management of the upper limb in children with spastic cerebral palsy (UPDATE). Cochrane Database Syst Rev 2010;(1):CD003469.

16. Sakzewski L, Ziviani J, Boyd R. Systematic review and meta-analysis of therapeutic management of upperlimb dysfunction in children with congenital hemiplegia. Pediatrics 2009;123:e1111-22.

17. Fehlings D, Rang M, Glazier J, Steele C. Botulinum toxin type A injections in the spastic upper extremity of children with hemiplegia: child characteristics that predict a positive outcome. Eur J Neurol 2001;8 Suppl 5:145-9.

18. Hanna SE, Rosenbaum PL, Bartlett DJ, Palisano RJ, Walter SD, Avery L, et al. Stability and decline in gross motor function among children and youth with cerebral palsy aged 2 to 21 years. Dev Med Child Neurol 2009;51:295-302.

19. Lee JS, Lee KB, Kim CW, Choi YN, Jung DH, Park SD, et al. Deformities of spine and lower extremities in teenagers with cerebral palsy. J Korean Acad Rehab Med 2008;32:135-42.

20. Miller F. Cerebral palsy. 1st ed. Wilmington: Springer; 2005.

21. Chen CM, Chen CY, Wu KP, Chen CL, Hsu HC, Lo SK. Motor factors associated with health-related qualityof-life in ambulatory children with cerebral palsy. Am J Phys Med Rehabil 2011;90:940-7. 